recommendations for people who have RA was highlighted in the qualitative study, and delivery preferences were identified. Intervention development was mapped to the Behaviour Change Wheel [3] and employed the Theory of Planned Behaviour as it's theoretical basis. The proposed intervention is outlined in Table 1.

Conclusions: We have developed a theory-based intervention which considers the preferences of key stakeholders. Future research will determine the feasibility and effectiveness of this intervention.

References:

[1] Larkin et al (2015) Behaviour change interventions to promote physical activity in rheumatoid arthritis: a systematic review. Rheum Intl 35(10):1631-40.

[2] Craig et al (2008) Developing and evaluating complex interventions: new guidance, BMJ (Clinical research ed.), 337, a1655.

[3] Michie et al (2014) The Behaviour Change Wheel: A Guide to Designing Interventions., Silverback Publishing: Great Britain.

Disclosure of Interest: None declared

DOI: 10.1136/annrheumdis-2017-eular.1374

\section{THU0739-HPR THE PHENOMENON OF ALEXITHYMIA IN SYSTEMIC LUPUS ERYTHEMATOSUS PATIENTS}

M. Ramkhelawon, R.A. Grekhoff, G. Suleymanova. Rheumatology, Research Institute for Clinical and Experimental Rheumatology, Volgograd, Russian Federation

Background: Alexithymia as a radical in the structure of premorbid personality is attracting the attention of researchers as one of the possible psychological risk factors in the development of psychosomatic disorders in rheumatic diseases. Clinical experience supports the evidence of the relevance of alexithymia conception in relation to the many somatic patients, who show a limited ability to describe and differentiate emotion and create fantasies, and hence indicate the role of alexithymia phenomenon in the pathogenesis of psychosomatic disorders in SLE.

Objectives: To study the relationship of the levels of alexithymia with other clinico-psychological characteristics.

Methods: 87 patients with systemic lupus erythematosus (SLE) was surveyed with the Toronto Alexithymia Scale (TAS)

Results: According to the results obtained, SLE patients demonstrated a high level of alexithymia $(73,86 \pm 2,6$ points). The results of the research showed that the level of alexithymia, on one hand, does not depend upon gender, age, character and activity of the pathological process $(p>0.05)$. On the other hand, there was a significant inverse correlation between alexithymia and the level of the patient's education $(r=-0,42 p=0.031)$. Moreover, internality in interpersonal interaction $(r=-0,44 p=0.028)$, and significant direct correlation with indicators such as, the disease duration $(r=-0,46$ at $p=0.01)$, the intensity of neurotic disorders (asthenia $(r=-0,42 p=0.033)$, depression $(r=-0,52 p=0.006)$, anxiety ( $r=0.43 p=0.028)$, hypochondriasis $(r=-0,48 p=0.01)$, as well as psychological defense mechanism "Regression" ( $r=0,42 p=0,022)$.

Conclusions: Therefore, between the development of alexithymia in SLE patients and certain clinico-psychological characteristics, a particular association exists which provides a definite step for psychotherapeutic interventions aimed at correcting alexithymia personality traits of SLE patients.

Disclosure of Interest: None declared

DOI: 10.1136/annrheumdis-2017-eular.2615

\section{THU0740-HPR INFLUENCE OF BIOFEEDBACK THERAPY ON THE MICRO-CIRCULATION IN SYSTEMIC SCLEROSIS}

M. Ramkhelawon, R.A. Grekhoff, G. Suleymanova, S.A. Kharchenko. Rheumatology, Research Institute for Clinical and Experimental Rheumatology, Volgograd, Russian Federation

Background: Microcirculatory disorders are one of the most important clinical symptoms of systemic sclerosis (SSc), therefore we found it feasible to evaluate the clinical efficacy of biofeedback (BFB) in the complex therapy of patients with SSc based upon analysis of nailfold capillaroscopy.

Objectives: To study the impact of the method of biofeedback therapy on microcirculation disturbances in patients with SSc.

Methods: The study included 40 patients with SSc under observation. Among the patients examined, $95 \%$ were women and $5 \%$ were men. The average age of the patients - $38 \pm 3,3$ years, duration of illness - 14 $\pm 2,6$ years. Raynaud's phenomenon (RP) was observed in all the patients. A severe form of RP was seen in $80 \%$ of the patients. The effectiveness of biofeedback therapy was analyzed by studying the dynamics of the measures from the nailfold capillaroscopy and comparing the data obtained of patients from the main and control groups.

Results: It was found that, patients who were under biofeedback therapy, showed significant positive dynamics in the following signs of capillaroscopic picture: dilation of the capillaries ( $c 2=9,643 p=0,026$ ), morphological changes of the capillaries ( $c 2=4,90 p=0,027$ ), and hemorrhage (c $2=4,514 p=0,034$ ). In the control group of patients, in only one indicator of capillaroscopy that a significant change was noted, particularly by the presence of dilated capillaries ( $c 2=5,833$ with $p=0,016$ ). These findings suggest that treatment results were significantly better in the main study group of patients with SSc.
Conclusions: The implementation of biofeedback therapy favors a decrease in reflex musculo-tonic syndromes, improvement of microcirculation and peripheral blood flow and significantly allows an amelioration in the results of SSc therapy.

Disclosure of Interest: None declared

DOI: 10.1136/annrheumdis-2017-eular.2616

\section{THU0741-HPR PLACEBO AND NOCEBO EFFECTS INDUCED BY CONTEXTUAL FACTORS. A SURVEY ON BELIEFS AND ATTITUDES OF ITALIAN MUSCULOSKELETAL PHYSIOTHERAPISTS}

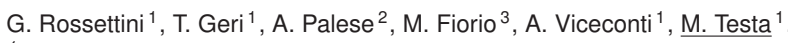
${ }^{1}$ Department of Neuroscience, Rehabilitation, Ophthalmology, Genetics, Maternal and Child Health, University of Genova, Savona; ${ }^{2}$ Department Biological and Medical Science, University of Udine, Udine, ${ }^{3}$ Department of Neurosciences, Biomedicine and Movement Sciences, University of Verona, Verona, Italy

Background: Context effects are well known and were described as integral part of the clinical setting (1). The conscious use of contextual factors (CF) has been recently proposed as an effective modality capable to influence the physiotherapy outcome by facilitation of placebo responses. (2) To date, the knowledge about CF adoption in clinical practice of physical therapists is absent.

Objectives: The aim of this study was to investigate frequency of use, beliefs and attitudes about contextual factors among Italian physical therapists specialized in manual therapy (OMTs)

Methods: An invitation to participate in an online survey was sent to 906 OMTs through the database of the Master in Musculoskeletal Rehabilitation (MRDM) of University of Genova by SurveyMonkey Software ${ }^{\circledR}$. These likely correspond to more than $85 \%$ of the whole OMTs population in Italy. A 17 items questionnaire and two clinical scenarios assessed behaviour, beliefs and attitudes of OMTs about CF adoption in clinical practice and data were analysed by descriptive statistic.

Results: 906 OMTs were invited to participate in the survey and 558 responded $(62 \%)$. The majority of OMTs uses CF in their practice frequently $(52 \%)$. They believe that an actual effect of CF can occur in acute pain $(57 \%)$, chronic pain $(78 \%)$ and rheumatologic disorders (56\%). OMTs consider the use of CF ethically acceptable when it exerts beneficial psychological effects and their effectiveness was shown during clinical experience $(31 \%)$. They disagree on the adoption of CF when they are based on deception, undermine the trust between OMT and patients, create legal problems or produce side effects (17\%). $38 \%$ of respondents do not communicate the use of CF to their patients and they adopt CF as addition to other physical therapy interventions with the aim of optimizing clinical responses $(20 \%)$. Expectation and psychological mechanisms are believed to be the main aspects behind placebo and nocebo effects induced by CF (7\%).

Conclusions: The use of CF is quite common among Italian OMTs and they mostly had positive attitudes towards their use and effectiveness.

References:

[1] Di Blasi Z, Harkness E, Ernst E, Georgiou A, Kleijnen J. Influence of context effects on health outcomes: a systematic review. Lancet. 2001 Mar 10;357(9258):757-62.

[2] Testa M, Rossettini G. Enhance placebo, avoid nocebo: How contextual factors affect physiotherapy outcomes. Man Ther. 2016;24:65-74.

Disclosure of Interest: None declared

DOI: 10.1136/annrheumdis-2017-eular.4361

\section{THU0743-HPR A NEW AND ALTERNATIVE TREATMENT IN SCLERODERMIC DIGITAL ULCERS}

M. Muratore ${ }^{1}$, D. Carati ${ }^{2}$, L. Quarta ${ }^{1}$, A. Grimaldi ${ }^{1}$, E. Quarta ${ }^{1}$, M. Garzya ${ }^{1}$, D. Longo ${ }^{1}$, O. Casilli ${ }^{1}$, F. Calcagnile ${ }^{1}$. ${ }^{1}$ U.O. Reumatologia- P.O. "V. Fazzi" Lecce, LECCE; ${ }^{2}$ Research \& development, Ekuberg Pharma, MARTANO, Italy

Background: Digital ulcers (DUs) are a major clinical problem for patients with systemic sclerosis (SSc). Patients with DUs may suffer from severe pain and often undergo a limitation of daily life activities, thus resulting in a functional impairment with a significant impact on the patient's health-related quality of life. Prevention of further complications and lesions is possible if the initial evaluation is performed early and correctly and if a treatment is started promptly.

Objectives: The primary objective is to demonstrate the effect of Lecoxen cream (Ekuberg Pharma, Italy) in comparison with another cream (Fitostimoline cream, DAMOR SpA, Italy) on the reduction of the number and size of DUs in patients with systemic sclerosis evaluating in addiction the reduction of pain dealing with DUs and quality of life.

Methods: In this single-blind randomized progressive trial 39 women, with confirmed digital ulcers present for at least 4 weeks with a surface area greater than $0.5 \mathrm{~cm}^{2}$ but smaller than $2,5 \mathrm{~cm}^{2}$, that follow lloprost therapy $(0,05 \mathrm{mg} / 2$ times/month), afferent to the Operative Unit (O.U.) of Rheumatology of the Local Health Unit (LHU) of San Cesario di Lecce, were randomized to receive the topical application of Lecoxen cream (group I: 20 women) or Fitostimoline cream (group II: 19 women). We took digital photographs to measure ulcer surface area and to draw the periwound area before the first cream application and after 30 days. Then an evaluation of DUs diameter and number was carried out. A 